\title{
Rate of imagery processing in two versus three dimensions
}

\author{
NANCY H. KERR \\ Oglethorpe University, Atlanta, Georgia
}

\begin{abstract}
A series of five experiments was conducted to test the optimal speed for performing two-and three-dimensional imagery tasks. Subjects were required to keep track of the location of a pathway in an imagined matrix, as the directions of its successive movements were described verbally. Matrices varied in size and in number of spatial dimensions, with two-dimensional matrices drawn on cardboard and three-dimensional ones built from wooden blocks. When subjects were able to dictate the rate of presentation of the terms describing the pathway, they preferred slower rates for three-dimensional than for two-dimensional stimuli. In subsequent experiments, very fast presentation rates had a larger detrimental effect on performance with three-dimensional matrices than with two-dimensional matrices. A comparison of the patterns of performance for subjects who generally scored high with the patterns for those who scored low showed a stronger effect of dimensionality for poor performers, suggesting that individual differences mediate performance on the task.
\end{abstract}

A variety of mental imagery tasks have provided ample evidence that people are capable of imagining transformations or movement in three-dimensional space (see, e.g., Kerr, 1987; Pinker, 1980; Pinker \& Kosslyn, 1978; R. N. Shepard \& J. Metzler, 1971). The theoretical accounts of underlying processes that mediate such imagery, however, vary widely. One central issue of debate has been whether two or three spatial dimensions are represented in the primary stage of imagery processing. In Kossyln's influential theory of imagery (Kosslyn, 1980; Kosslyn \& Shwartz, 1977), the initial stage of both visual perception and visual imagery involves the activation of a two-dimensional array of cells that correspond to specific regions of the visual field. Objects and scenes are represented in the array by patterns of filled cells that are responsive to information in the visual field, or, in the case of imagery, information from long-term memory. Information about the third dimension is not coded directly, but must be extracted from the two-dimensional representation. In sharp contrast are theories that posit that the primary medium of mental imagery is inherently three-dimensional (see, e.g., Attneave, 1972; R. N. Shepard, 1981, 1984). These "sandbox in the head" (Attneave, 1972) theories propose that depth is encoded directly in perceiving and imagining, and that no special status is afforded to the picture plane or any other twodimensional surface. One area of conflict for these dif-

I am grateful to Lawrence Barsalou and Jamie Medowar for their valuable assistance in designing and conducting the first experiment, to Laura Bradley and Michael Bohannon for their assistance in statistical analyses, and to David Foulkes for his suggestions on earlier drafts of the manuscript. Comments by Cesare Cornoldi, Pierre Jolicoeur, and an anonymous reviewer were very helpful in the revision of the manuscript. Correspondence should be sent to N. H. Kerr, Oglethorpe University, 4484 Peachtree Road, N. E., Atlanta, GA 30319. fering theoretical views consists in their predictions about the amount of time required to perform imagery tasks based on two- versus three-dimensional stimuli. "Array" theories generally predict longer processing times for three-dimensional imagery tasks, whereas "sandbox" theories do not.

At an empirical level, the primary arena for testing the conflicting theoretical predictions has been the mental rotation task first reported by R. N. Shepard and J. Metzler (1971). Their original finding was that the linear reaction time function relating degrees of rotation between two drawings of three-dimensional figures and time required to judge whether they were identical was essentially the same whether the figures had been rotated on the picture plane or in depth. The authors interpreted their results as evidence that the two-dimensional projection of the drawing of a three-dimensional figure has little relevance for the mental rotation process, which occurs in a threedimensional medium (J. Metzler \& R. N. Shepard, 1974). However, others have argued that the results were due to the fact that the three-dimensional stimuli were so convincingly drawn in depth that subjects did not recognize the possibility of using a picture-plane rotation strategy (Shwartz, 1981). Thus, they encoded and rotated all stimuli in three-dimensional space and never considered mentally rotating the picture itself on a two-dimensional plane. Subsequent research has compared rotation rates of drawings of three-dimensional stimuli with those of drawings of two-dimensional stimuli. The results have been mixed, with some researchers emphasizing differences in rotation rates (e.g., Shwartz, 1981; Jolicoeur, Regehr, Smith, \& Smith, 1985), and others, similarities (Cooper \& Farrell, described in R. N. Shepard \& Cooper, 1982, pp. 178-181; S. Shepard \& D. Metzler, 1988). In the long run, the rotation task may prove incapable of providing conclusive 
evidence in comparisons of two- and three-dimensional imagery processing because, as S. Shepard and D. Metzler (1988) note, it is difficult if not impossible to equate drawings of two- and three-dimensional objects on other relevant dimensions such as complexity.

The research reported here was designed to test time constraints in two- and three-dimensional imagery processing by using another imagery task, in which subjects imagined symmetrical matrices that served as a stable framework for a series of mental manipulations or transformations described to them verbally. Similar tasks have been used to establish the modality-specific nature of imagery processing (Brooks, 1967), to determine characteristics of imaging that are similar to those of perceiving (M. J. Peterson, 1975), and to test processing capacity on the basis of the total number of units in a matrix (Attneave \& Curlee, 1983; Kerr, 1987), as well as the number of units that must be held in memory for immediate report (L. R. Peterson, Rawlings, \& Cohen, 1977). Like the mental rotation tasks, these experiments have been shown to involve primarily spatial aspects of mental imagery, rather than purely visual ones (Farah, Hammond, Levine, \& Calvanio, 1988). The advantages of this paradigm are these: (1) performance on the task is relatively invulnerable to explanations based on mental processes other than visuospatial ones or on artifacts such as experimenter expectations (Intons-Peterson, 1983); (2) threedimensional stimuli can be fully constructed rather than depicted in drawings; (3) it is possible to control and manipulate a variety of task variables with precision; (4) twoand three-dimensional matrices can be matched for complexity as defined either by the total number of units constituting the matrix $(8 \times 8 ; 4 \times 4 \times 4)$ or the number of units per dimension $(4 \times 4 ; 4 \times 4 \times 4)$; and (5) of special relevance to the research reported here, the rate of imagery processing can be both measured as a dependent variable and manipulated as an independent variable.

The specific matrix task employed here was adapted from Kerr (1987). In it, subjects are shown symmetrical matrices of varying sizes that have been drawn on cardboard (e.g., $3 \times 3,4 \times 4$ ) or constructed from wooden blocks $(3 \times 3 \times 3,4 \times 4 \times 4)$. On a single trial of the experiment, the subject must imagine a particular matrix with one of its units (a square or a block) designated as the starting place for a pathway that moves from unit to unit through the matrix. The direction of each successive step in the pathway is then dictated by the sequential auditory presentation of the terms left, right, up, down (back, front). When the description of the pathway has ended, the subjects are asked to indicate the location of the final step in the pathway. The subjects are given repeated trials with each matrix, and performance is measured by the number of correct trials. In the first experiment reported here, the subjects were allowed to dictate the rate of presentation of terms describing the pathways so that the experimenter could assess preferred presentation rates for matrices that varied both in the number of units they contained and in whether they were two- or three-dimensional. In subsequent experiments, presentation rate was varied as an independent variable, to test the effects of differing rates on task performance.

In addition to allowing a test of general processing time requirements for two- and three-dimensional imagery tasks, the present design allows a test of a different theoretical question regarding the possibility for incorporation in an image of items that are occluded or "hidden" from direct view. Array theories preclude the presence of any objects in an image that cannot be seen from the subject's vantage point, whereas sandbox theories do not. Previous research on incidental memory for items that have been described in concealed or hidden locations of an imagined scene has produced mixed results, with some researchers reporting equivalent memory for visible and hidden aspects of an image (Kerr \& Neisser, 1983; Neisser \& Kerr, 1973), and others reporting poorer recall for concealed items (Keenan \& Moore, 1979). The theoretical motivation for the original hidden imagery experiment (Neisser \& Kerr, 1973) provides a third alternative to those just discussed. On the basis of J. J. Gibson's $(1966,1979)$ theory of perception, Neisser and I proposed that imaging, like perceiving, incorporated direct information about a layout and that the process was extended in time. Thus, the finding that images that included hidden items were recalled as well as those in which an item was visible was interpreted as consistent with Gibson's view that "perception is not limited to the surfaces that happen to be visible at a given instant; one perceives the entire layout, including objects that are temporarily concealed" (Kerr \& Neisser, 1983, pp. 212213). The finding that creation of images that included hidden items took more time than did those that were visible was attributed to the greater complexity of spatial relationships in three-dimensional layouts.

In the present experimental design, subjects were asked to imagine pathways that moved through blocks, some of which could not be seen from the subject's vantage point. In a $3 \times 3 \times 3$ figure, for example, only the blocks that constitute the front and top of the figure can be directly perceived by the subject. The blocks on the sides of the figure could be perceived by a moderate change in perspective, but the blocks in the center and back of the figure are quite thoroughly occluded from view. If array theories are right, pathways that include these hidden blocks should produce more errors than those that do not.

A secondary focus of the research reported here was on individual differences in the ability to perform the matrix task. Cornoldi, Cortesi, and Preti (1991) selected groups of subjects high and low in visuospatial skills as assessed by a standard paper and pencil test, and they evaluated their ability to perform the Kerr (1987) pathwaymatrix task with the following figures: $3 \times 3,2 \times 2 \times 2$, $4 \times 4,3 \times 3 \times 3,8 \times 8$, and $4 \times 4 \times 4$. In general, high visuospatial scorers performed the task with fewer errors than did low visuospatial scorers, but there was also a difference in the pattern of results for the two groups. The 
results for high visuospatial scorers essentially replicated those reported by Kerr (1987) with nearly perfect performance for the $3 \times 3,2 \times 2 \times 2$, and $3 \times 3 \times 3$ figures, significantly lower performance on the $4 \times 4$, and still lower performance on the $8 \times 8$ and $4 \times 4 \times 4$ figures. In contrast, low visuospatial scorers did not show the advantage in performance on the $3 \times 3 \times 3$ over performance on the $4 \times 4$ matrix, and performance was significantly poorer with the $4 \times 4 \times 4$ matrix than with the $8 \times 8$ or any other matrix. The authors speculated that the shift from two to three dimensions in an imagery task may increase the processing demands beyond the capabilities of subjects with poor visuospatial skills but not beyond those of subjects with good skills. These results are consistent with Just and Carpenter's (1985) report of differences between high and low visualizers in the strategies that they adopted to perform three-dimensional imagery tasks. Although the experiments reported here were not designed as tests of individual differences, some of the studies included a sufficient number of subjects of varying overall ability on the tasks to permit an independent assessment of Cornoldi et al.'s findings, with overall scores on the matrix task used to select subjects assumed to be high and low in visualizing ability. Without an independent assessment of visualization abilities and comparison measures of other abilities (e.g., verbal), the assumption that visualization ability mediates performance on the matrix task remains tentative. Nevertheless, Cornoldi et al.'s findings add to its plausibility.

Experiment 1 was computer controlled, with pathway directions presented visually on the computer screen. Because reading has been shown to interfere with performance on similar tasks (Brooks, 1967), pilot testing was conducted to determine whether performance would be impaired if subjects read directions themselves rather than heard them read by the experimenter. Pilot subjects reported no difficulty in responding to visually presented directions, and their response times were faster and less variable than those of subjects who heard the directions read by the experimenter. Visual presentation was therefore adopted for the formal experiment. The design of Experiment 1 included only the matrices on which performance had previously been nearly perfect with a 2-sec presentation rate (Kerr, 1987). The two-dimensional figures were $2 \times 2$ and $3 \times 3$, and the three-dimensional matrices were $2 \times 2 \times 2$ and $3 \times 3 \times 3$, allowing two comparisons based on the number of units per spatial dimension $(2 \times 2$ vs. $2 \times 2 \times 2 ; 3 \times 3$ vs. $3 \times 3 \times 3)$ and one based on approximately equivalent numbers of units distributed in two- versus three-dimensional space $(3 \times 3$ vs. $2 \times 2 \times 2)$.

\section{EXPERIMENT 1}

\section{Method}

Subjects. Twelve female and 12 male undergraduates at Emory University participated in partial fulfillment of a psychology course requirement.

Materials. The $2 \times 2$ and $3 \times 3$ matrices were drawn in black ink on white cardboard. Each square in the matrix was $16 \mathrm{~cm}^{2}$, result- ing in a total size of $64 \mathrm{~cm}^{2}$ for the $2 \times 2$ matrix and $144 \mathrm{~cm}^{2}$ for the $3 \times 3$ matrix. The $2 \times 2 \times 2$ and a $3 \times 3 \times 3$ matrices were built with wooden blocks, each of which measured $64 \mathrm{~cm}^{3}$, for total figure sizes of $512 \mathrm{~cm}^{3}$ and $1,728 \mathrm{~cm}^{3}$, respectively.

For each matrix, 48 different pathways, each consisting of a starting square or block and seven statements of direction, were randomly generated, with the stipulation that no directional term appear more than twice consecutively. The directional terms were up-down, left-right, and, for the three-dimensional figures, forward-backward. The directional terms were displayed, at eye level, on a North Star computer screen 3-4 m from the subject.

Design and Procedure. The subjects were given instructions and two demonstration trials with each matrix, in the order $2 \times 2,3 \times 3$, $2 \times 2 \times 2,3 \times 3 \times 3$. They were instructed to perform the task as quickly as possible without sacrificing accuracy. Next, a standard set of 20 practice trials, 5 per matrix in random order, was administered, followed immediately by 48 experimental trials, 12 per matrix, randomly selected from the pool of 48 and randomly ordered. Experimental trials on which errors occurred were replaced with randomly selected trials from the same source.

A trial began when the experimenter pointed to one of the matrices to indicate the relevant matrix and its starting square or block. When blocks were not directly visible from the subject's vantage point, the starting block was indicated by a combination of pointing and verbal description (e.g., the block directly below this one), or by moving the blocks to allow direct pointing. All matrices were then hidden from view. The experimenter initiated the computergenerated directional terms, the first of which was preceded by a warning tone and the presentation of an asterisk (*) on the screen. Each subsequent term appeared at a $300-\mathrm{msec}$ delay after the subject pressed a key to indicate readiness for it. At the end of the series, the experimenter again uncovered the matrix and asked the subject to indicate the final square or block in the pathway, by pointing, verbal description, or a combination of the two.

\section{Results}

The average selected presentation rates for the four conditions are presented in Figure 1. Subjects identified the correct final point in a pathway with $94 \%$ accuracy on trials with the $2 \times 2$ matrix, $91 \%$ for both the $3 \times 3$ and $2 \times 2 \times 2$ matrices, and $80 \%$ for the $3 \times 3 \times 3$ matrix.

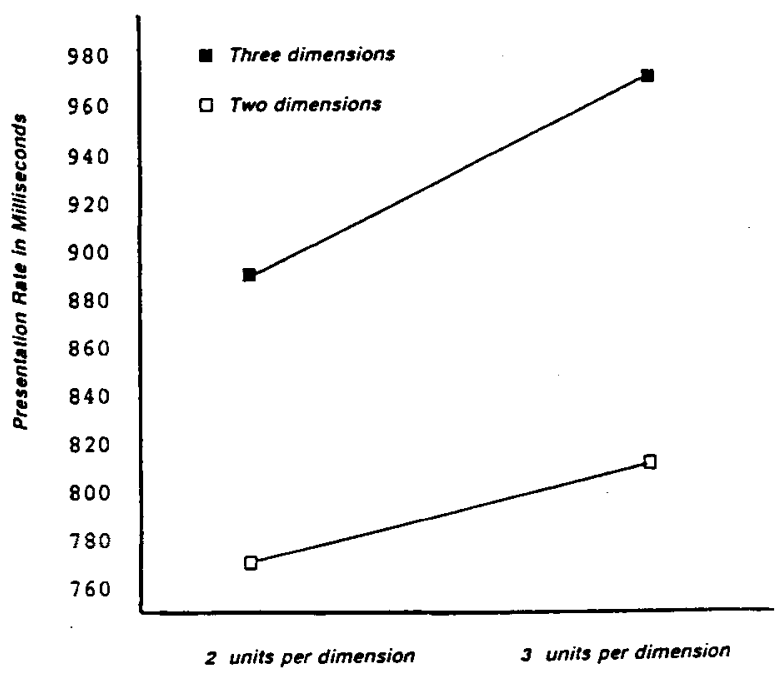

Figure 1. Average preferred presentation rates for matrices in Experiment 1. 
A $2 \times 2$ within-subject analysis of variance (ANOVA) of presentation rate revealed significant main effects of number of dimensions (two vs. three) $[F(1,23)=24.07$, $\left.M S_{\mathrm{e}}=19,426, p<.001\right]$ and matrix size (2 units per dimension vs. 3) $\left[F(1,23)=13.88, M S_{\mathrm{e}}=7,286, p<\right.$ $.005]$, but the interaction was not significant $[F(1,23)=$ $\left.0.26, M S_{\mathrm{e}}=24,206\right]$. The additional planned comparison of the $3 \times 3$ with the $2 \times 2 \times 2$ matrix was also significant, using Bonferroni criteria at the .05 level. A $2 \times 2$ within-subject ANOVA of the number of incorrect trials with each figure yielded significant effects of number of dimensions $\left[F(1,23)=13.53, M S_{\mathrm{e}}=1.49, p<.005\right]$ and matrix size $\left[F(1,23)=7.65, M S_{\mathrm{e}}=2.40, p<.025\right]$, as well as a significant interaction $\left[F(1,23)=3.78, M S_{\mathrm{e}}\right.$ $=1.59, p<.01]$.

The subjects' overall ability to perform the task, as assessed by total number of correct trials across all matrices, varied considerably, with a range of $67 \%-100 \%$ correct. The total number of correct trials was used as a criterion for comparing patterns of performance of subjects of differing overall task ability. Dividing the subjects in thirds on the basis of overall performance produced a highscoring group of 8 subjects who performed with an average of $97 \%$ accuracy, and a low group of 8 who scored $80 \%$ on the average. Half the subjects in each subgroup were female. A summary of accuracy and presentation rate data for these two subgroups is presented in Table 1. An ANOVA of presentation rate with these data showed significant main effects of number of dimensions $[F(1,14)$ $\left.=47.12, M S_{\mathrm{e}}=4,412.79, p<.001\right]$ and number of units $\left[F(1,14)=21.06, M S_{\mathrm{e}}=2,230.42, p<.001\right]$, but not of group. No interaction was significant.

\section{Discussion}

The presentation rates selected by subjects were responsive both to the number of units per dimension in a matrix and to the number of spatial dimensions in which these units were distributed. The total number of units in a matrix appeared to be less important, as is evidenced by the slower times selected for the matrices with 8 blocks than for those with 9 squares and by the equivalent decrease in rate selected for larger figures in two and three dimensions despite the much greater increase in number of component units for the three-dimensional matrices.

Data based on accuracy showed little variability for the three smallest matrices but a sharp drop in performance for the $3 \times 3 \times 3$ matrix, as indicated by the significant interaction. This finding is inconsistent with Kerr's (1987) results, but compatible with several of the findings re- ported by Cornoldi et al. (1991). The data summary based on high and low scorers provides additional support for Cornoldi et al.'s explanation of differences between Kerr's results and theirs. There are clear individual differences in the ability to perform the task, with apparent ceiling effects for high scorers. Cornoldi et al.'s high school educated subjects may have had generally poorer visuospatial skills than Kerr's college samples.

Interestingly, the high scorers selected faster presentation rates for all matrices than did the low scorers. $\mathrm{Al}$ though this difference was not statistically significant, nevertheless its direction bolsters the interpretation of performance differences based on general task performance ability rather than an artifact such as a speed-accuracy tradeoff. Overall patterns of preferred rates were similar for the two subgroups. Somewhat surprisingly, the increments in preferred rates were also similar. Despite the low scorers' difficulty with the $3 \times 3 \times 3$ matrix, as indicated by poor accuracy, the presentation rates that they selected were no slower in relation to the rates for smaller matrices than were those of the high scorers. Although this finding could be attributed to a speed-accuracy tradeoff, Cornoldi et al.'s finding of poor performers' difficulty with the $3 \times 3 \times 3$ matrix at a 2 -sec presentation rate makes this possibility seem unlikely. Presentation rate appears not to be the primary source of difficulty for poor performers on the matrix task.

Experiment 2 was designed as an initial test of the consequences of very rapid presentation rates on performance of the matrix task. Presentation rates of 1 and $.5 \mathrm{sec}$ were selected to be, respectively, slightly slower and slightly faster than the average rates favored in Experiment 1 . Three matrices were included, $3 \times 3,2 \times 2 \times 2$, and $3 \times 3 \times 3$, to permit comparisons based on equivalent numbers of units per dimension $(3 \times 3,3 \times 3 \times 3)$ and on roughly equivalent total numbers of units $(2 \times 2 \times 2,3 \times 3)$.

\section{EXPERIMENT 2}

\section{Method \\ Subjects. Eight female and 10 male Oglethorpe University under- graduates participated for extra credit in a psychology class. \\ Materials. The $3 \times 3,2 \times 2 \times 2$, and $3 \times 3 \times 3$ matrices were the same as those used in Experiment 1. Forty-eight different pathways were generated according to the same procedure as in Experiment 1. Six tape recordings were made for presentation of the directional terms, each with six blocks of eight trials, four presented at the fast rate (directions read every $.5 \mathrm{sec}$ ) and four at the slow rate (directions read every $1 \mathrm{sec}$ ). The first three blocks included each of the three matrices in a different order for each tape, and the sec-}

Table 1

Mean Percent Correct and Preferred Presentation Rate (PR, in Milliseconds) for Two Subgroups in Experiment 1

\begin{tabular}{|c|c|c|c|c|c|c|c|c|}
\hline \multirow[b]{3}{*}{ Subgroup } & \multicolumn{8}{|c|}{ Matrix } \\
\hline & \multicolumn{2}{|r|}{$2 \times 2$} & \multicolumn{2}{|r|}{$3 \times 3$} & \multicolumn{2}{|c|}{$2 \times 2 \times 2$} & \multicolumn{2}{|c|}{$3 \times 3 \times 3$} \\
\hline & PR & $\%$ Correct & PR & \% Correct & PR & \% Correct & PR & $\%$ Correct \\
\hline High scorers* & 690 & 100 & 719 & 98 & 777 & 98 & 834 & 92 \\
\hline Low scorers* & 769 & 89 & 845 & 82 & 907 & 82 & 961 & 66 \\
\hline
\end{tabular}

$*_{n}=8$. 
ond three blocks presented the matrices in reverse order, with the order of fast and slow trials also reversed. For example, Tape 1 included blocks of four trials each in the order $3 \times 3$ slow, $3 \times 3$ fast, $2 \times 2 \times 2$ slow, $2 \times 2 \times 2$ fast, $3 \times 3 \times 3$ slow, $3 \times 3 \times 3$ fast, $3 \times 3 \times 3$ fast, $3 \times 3 \times 3$ slow, $2 \times 2 \times 2$ fast, $2 \times 2 \times 2$ slow, $3 \times 3$ fast, $3 \times 3$ slow. Each of the 48 pathways appeared on two tapes, once at the fast rate and once at the slow rate.

Procedure. The subjects were given instructions and sample pathways for each of the three matrices while the figures were physically present. They were then given 2 slow and 2 fast practice trials, with each figure identical to that on the experimental trials except that feedback was given. Each trial began with the experimenter's pointing to or describing the location of a starting square or block on a matrix, which was then covered as the directions were read on tape. The matrix was exposed at the end of the trial, so the subject could indicate the final block. The practice trials were followed by 48 experimental trials presented on one of the six tape recordings. The subjects were told whether the presentation rate would be fast or slow prior to each block of four trials at that rate.

\section{Results}

The results are summarized on the first two lines of Table 2. A two-way ANOVA with three kinds of matrices and two presentation rates revealed significant effects of the matrix variable $\left[F(2,34)=7.24, M S_{\mathrm{e}}=1.60, p<\right.$ $.005]$ and presentation rate $\left[F(1,17)=31.38, M S_{\mathrm{e}}=\right.$ $2.39, p<.001]$, as well as a significant interaction between them $\left[F(2,34)=9.56, M S_{\mathrm{e}}=0.91, p<.001\right]$. Bonferroni comparisons at the .05 level were significant for the contrast of the $3 \times 3$ with the $3 \times 3 \times 3$, but not for either comparison with the $2 \times 2 \times 2$.

As in Experiment 1, the subjects were divided in thirds on the basis of the total number of correct trials, in order to compare the patterns of performance of subjects who differed in levels of ability to perform the task. The results, based on the 6 highest and lowest scorers, are presented on the last four lines of Table 2. Four of the highest scorers and 3 of the lowest were female. A three-way ANOVA of these data yielded the expected significant main effects of group $\left[F(1,10)=68.44, M S_{\mathrm{e}}=0.99\right.$, $p<.001]$, matrix $\left[F(2,20)=4.27, M S_{\mathrm{e}}=1.54, p<\right.$ $.05]$, and presentation rate $\left[F(1,10)=270.00, M S_{\mathrm{e}}=\right.$ $0.15, p<.001]$. Also significant were the interactions between group and presentation rate $[F(1,10)=213.33$, $\left.M S_{\mathrm{e}}=0.15, p<.001\right]$ and between matrix and presen-

Table 2

Mean Percent Correct at Fast and Slow Presentation Rates for Each Matrix in Experiment 2

\begin{tabular}{cccc}
\hline & \multicolumn{3}{c}{ Matrix } \\
\cline { 2 - 4 } Rate & $3 \times 3$ & $2 \times 2 \times 2$ & $3 \times 3 \times 3$ \\
Slow & \multicolumn{3}{c}{ All Subjects } \\
Fast & 94 & 95 & 92 \\
& 84 & 76 & 58 \\
Slow & \multicolumn{4}{c}{ High Scorers* } \\
Fast & 96 & 98 & 96 \\
& 98 & 98 & 88 \\
Slow & \multicolumn{4}{c}{ Low Scorers* } \\
Fast & 88 & 90 & \\
\hline$*$
\end{tabular}

tation rate $\left[F(2,20)=12.62, M S_{\mathrm{e}}=0.72, p<.001\right]$, as well as their three-way interaction $[F(2,20)=5.29$, $\left.M S_{\mathrm{e}}=0.72, p<.05\right]$. Bonferroni comparisons were again significant only for the $3 \times 3$ and $3 \times 3 \times 3$ matrices.

For the purposes of identifying pathways in the $3 \times 3 \times 3$ matrix that included hidden blocks, the 15 blocks on the front and top of the figure were considered "visible" from the subject's point of view, the four remaining blocks on each side of the figure were considered "potentially visible" with a moderate shift in vantage point, and the final four blocks in the middle and back of the figure were considered "hidden." The average accuracy on pathways that included at least one hidden block was $77 \%$, and the average was $74 \%$ for pathways that included no hidden blocks. The average based only on the subset of pathways that were always directly visible (no "side" blocks) was $75 \%$, and for two pathways that began and remained on the front surface of the figure (moving only up, down, left, and right) the average was $58 \%$.

\section{Discussion}

A faster presentation rate than the self-paced rates in Experiment 1 produced decrements in performance for all three matrices, in comparison with the performance at the slower rates. In addition, whereas performance with all three matrices was similarly high at the slow rate, performance differences appeared at the fast rate, with greatest accuracy with the $3 \times 3$ matrix and worst performance with the $3 \times 3 \times 3$. Thus, the results confirm previous observations that adding a third dimension can increase imagery processing capacity at slow presentation rates (Kerr, 1987), but they also show that the advantages of increased capacity are limited. At faster presentation rates, the advantage disappears. The intermediate performance levels with the $2 \times 2 \times 2$ matrix suggest that both the absolute number of units and the number of dimensions in which the units are distributed may be important determinants of task performance. However, the failure of this figure to differ significantly from the other two limits interpretations based on it.

Individual differences in ability to perform the task apparently mediated sensitivity to the independent variables. Performance by low overall scorers was markedly more affected by both the matrix and the presentation rate than was that of high scorers, as is indicated by the three-way interaction. As in Experiment 1, there were apparent ceiling effects for the high scorers.

The analysis of pathways in which some blocks in the $3 \times 3 \times 3$ figure were hidden from the subject's view provided no evidence that the presence of these blocks made the task more difficult. In fact, subjects made slightly fewer errors with these pathways than they did with visible ones. Two pathways that remained on the front surface of the matrix, and thus were equivalent to pathways in a $3 \times 3$ matrix according to "occlusion" criteria, did not show the nearly perfect performance that would be predicted were occlusion the cause of difficulty.

The finding that a three-dimensional imagery task requires more time than a two-dimensional one is consis- 
tent with array theories of imagery, as well as with the Gibsonian theory that motivated earlier research with hidden imagery. However, the relevance of the depth dimension for the two theories is quite different. In array theories, depth is a secondary quality of imagery that is inferred from the primary two-dimensional array. In Gibson's approach, depth has no special, "added-on" status: it is perceived directly. Thus, array theories would predict slower imagery processing times whenever the depth dimension was included in a matrix, even if the matrix were itself essentially two-dimensional (e.g., a $3 \times 3$ matrix that was only one unit wide, but three units high and three units deep). The Gibson-based theory predicts longer processing times only when all three dimensions must be included.

Experiment 3 was designed as a test of the differing predictions from these two theoretical points of view. Five matrices built from wooden blocks were tested in a paradigm similar to that of Experiment 2, including both previous three-dimensional matrices and three "twodimensional" matrices that were constructed from nine blocks each in the form of a $3 \times 3$ display. The pictureplane matrix faced the subject directly so that pathways were described with the terms left, right, up, and down; the tall-depth matrix stood upright facing away from the subject so that pathways were described with the terms up, down, backward, and forward; the flat-depth matrix faced flat down so that pathways were described with the terms backward, forward, left, and right. All pathway directions were read at the fast $(.5$-sec) speed because only at this rate had two- versus three-dimensional differences in accuracy occurred. If poorer performance with threedimensional stimuli is due specifically to the inclusion of the depth dimension, performance should be superior for the figure that omits depth (the picture-plane $3 \times 3$ ). However, if number of dimensions, regardless of depth, is the relevant variable, all $3 \times 3$ figures should be easier than the matrices built in three dimensions.

\section{EXPERIMENT 3}

\section{Method}

Subjects. Twelve female and 8 male Oglethorpe University students participated for extra credit in an introductory psychology class.

Materials. Forty pathways were randomly generated as in the previous experiments for the $2 \times 2 \times 2,3 \times 3 \times 3$, and $3 \times 3$ pictureplane matrices. Forty pathways for the other two $3 \times 3$ matrices were constructed by substituting the terms backward and forward for left, right (tall-depth matrix), or up, down (flat-depth matrix) in the previously generated $3 \times 3$ pathways. Five tape recordings were made of the pathways in blocks of eight trials per figure presented at a .5 -sec rate. Each matrix appeared once in each serial position on tape, and the $3 \times 3$ matched pathways were counterbalanced across tapes.

Procedure. Each matrix was introduced just prior to the block of trials in which it was tested. The task was described in detail for the first matrix and in shortened form for each subsequent matrix. Two practice trials with feedback were presented on tape prior to each block of experimental trials. The procedure was otherwise identical to that in the previous experiments.

\section{Results}

Mean percentages of correct trials are presented in the first line of Table 3. A one-way ANOVA showed a significant effect $\left[F(4,76)=12.71, M S_{\mathrm{e}}=1.90, p<.001\right]$. Newman-Keuls pairwise comparisons at the .05 level showed performance with the $3 \times 3 \times 3$ to be significantly poorer than that with any other figure, performance with the $2 \times 2 \times 2$ to be significantly worse than that with the $3 \times 3$ picture-plane matrix, but no other significant differences.

Once again, large individual differences in overall accuracy permitted a comparison of performance patterns based on the scores of the highest and lowest scorers ( $n=7$ in each group). These data are presented in the second and third lines of Table 3. Two of the highest and 5 of the lowest scorers were female. A two-way ANOVA confirmed the significant effects of group $[F(1,12)=$ $\left.157.94, M S_{\mathrm{e}}=1.53, p<.001\right]$ and matrix $[F(4,48)=$ 9.78, $\left.M S_{\mathrm{e}}=1.45, p<.001\right]$, as well as a significant interaction between them $\left[F(4,48)=4.41, M S_{\mathrm{e}}=1.45\right.$, $p<.005]$.

\section{Discussion}

The results reinforce the finding from Experiment 2 that at fast presentation rates, imaginal processing of threedimensional figures is no longer equivalent to that of twodimensional stimuli, and the larger three-dimensional figure suffers especially. The failure to find statistically significant differences among the three $3 \times 3$ figures suggests that the depth dimension itself plays no role in the difficulty that subjects experience with three-dimensional figures. However, three aspects of the data might be interpreted as evidence in favor of a special status for the depth dimension. First, performance with the picture-plane figure was better than that with the other two, even though not significantly so. Second, the picture-plane figure was the only one significantly superior to the $2 \times 2 \times 2$. And third, performance by the lowest scorers on the task, who may be most sensitive to task difficulty, shows strong superiority for the picture-plane matrix over the other two $3 \times 3$ matrices.

Comments from subjects suggested a possible artifactual explanation for any apparent superiority of the picture-plane matrix. The terms for directional moves in depth, backward and forward, were the only two-syllable terms used, and their presentation necessarily diminished the interstimulus interval between terms. For some sub-

Table 3

Mean Percent of Correct Trials With the 5 Matrices in Experiments 3 and 4

\begin{tabular}{cccccc}
\hline & \multicolumn{5}{c}{ Matrix } \\
\cline { 2 - 6 } & $3 \times 3 *$ & $3 \times 3 \dagger$ & $3 \times 3 \ddagger$ & $2 \times 2 \times 2$ & $3 \times 3 \times 3$ \\
\hline Experiment 3 & 82 & 74 & 72 & 64 & 46 \\
High scorers & 96 & 96 & 93 & 82 & 82 \\
Low scorers & 80 & 46 & 38 & 50 & 14 \\
Experiment 4 & 69 & 71 & 58 & 48 & 33 \\
\hline
\end{tabular}

*Picture plane. †Tall depth. $\ddagger$ Flat depth. 
jects, this created the illusion of faster rates when the twosyllable terms were included. In addition, the terms backward and forward had potentially ambiguous meanings. In the experiment, they were used to indicate forward movement toward the front of the figure closest to the subject or backward movement toward the more distant part of the matrix. This usage conflicted with a more salient meaning for some subjects, in which the terms referred to one's own movement forward or backward in locomotion through space.

Experiment 4 was designed to clarify the results of Experiment 3 by substituting the directions front and back for those previously used to indicate moves in depth. These single-syllable terms had clear reference to the matrix, not the viewer. The design of Experiment 3 was otherwise replicated.

\section{EXPERIMENT 4}

\section{Method}

Subjects. Fifteen (14 female and 1 male) graduate students in education at Oglethorpe University volunteered to participate as part of a classroom demonstration.

Materials. Except for the substitution of the terms front, back for forward, backward, the materials were identical to those for Experiment 3.

Procedure. Because it was necessary to complete the experiment within a single 3-h class period, the procedure was altered slightly. All 15 subjects received instructions and practice trials with all five matrices at the beginning of the class. In addition, the subjects were taught the coding system used by the experimenter to record starting and ending blocks in writing. It is a simple code in which blocks are numbered consecutively and consistently across the figures, and it had been learned easily and used accurately by subjects in pilot testing. When all subjects had mastered the task and the code, they participated in the experiment in groups of 3, with each group assigned to one of the five presentation tapes. As before, each block of eight experimental trials was preceded by two practice trials with feedback. The experimenter began each trial by pointing to the starting block and naming its numerical code. She then covered the matrix while the directions were read on tape, and she exposed it again as subjects each recorded the code for the final block on an answer sheet.

\section{Results}

The mean percent correct for each figure is presented on the fourth row of Table 3. A one-way ANOVA showed a significant effect $\left[F(4,56)=11.34, M S_{\mathrm{e}}=2.05, p<\right.$ $.001]$. Newman-Keuls comparisons at the .05 level showed performance with the $3 \times 3 \times 3$ to be significantly lower than performance with any other matrix and performance with the $2 \times 2 \times 2$ to be significantly lower than that with the picture-plane and tall-depth $3 \times 3$ matrices. Performance did not differ significantly among the $3 \times 3$ matrices.

The subjects' overall performance in this experiment was considerably lower than that in Experiment 3, and there was no group of "high scorers" comparable to those in the previous experiments. Consequently, comparisons of high and low scorers were not possible.

With the use of the same criteria as in Experiment 2, the combined data from the $3 \times 3 \times 3$ matrices in Experi- ments 3 and 4 were summarized on the basis of whether pathways included hidden blocks. An average of $40 \%$ correct trials occurred when pathways included a hidden block, and $43 \%$ when they did not. Two pathways that began and remained only on the front surface of the figure had an accuracy of $64 \%$, and one pathway that moved through hidden blocks for all but two steps of the pathway had an accuracy of $71 \%$.

\section{Discussion}

The results of Experiments 3 and 4 indicate that imagining the pathways in three-dimensional figures is more difficult than imagining them in two-dimensional figures at fast presentation rates, and that this may be true even when matrices are roughly matched for the total number of units $(2 \times 2 \times 2$ vs. $3 \times 3)$. The failure to find significant differences among the $3 \times 3$ figures in both experiments indicates that the difficulty with the threedimensional figures is due to the number of spatial dimensions, and not specifically to the depth dimension. The contribution of individual differences to task performance was especially apparent in these two experiments, both in the comparison of results from the two experiments, and in the comparison of the high and low scorers in Experiment 3.

Although it was not significant in either experiment, there was a tendency in both for poorer performance with the flat depth matrix than with the other two $3 \times 3 \mathrm{ma}-$ trices. One possible explanation is that this figure was not clearly perceived to recede in depth as it lay on the table top, and thus the front and back directions were difficult to translate into movements. Cornoldi et al. (1991) used an identical figure, but they employed the terms up-down instead of back-front. For people accustomed to reading books as they lie on flat surfaces, these directions might be more natural.

Once again, the pathways in the $3 \times 3 \times 3$ matrix that included hidden blocks were no more difficult for subjects than were the pathways without them. The two matrices that remained on the front of the figure had fewer than the average number of errors predicted by occlusion theories, but so did one pathway with a large proportion of hidden steps, which according to those theories should have been especially difficult.

The experiments reported so far have been limited to an examination of the effects of presentation rate on matrices with which subjects performed very well at slow rates in previous studies (Kerr, 1987). Experiment 5 was designed as a test of the effects of presentation rate on larger matrices with which subjects have experienced difficulty even at slow presentation rates. The design included three variables: size (defined by the absolute number of squares or blocks), presentation rate $(0.5 \mathrm{sec}, 1.0 \mathrm{sec})$, and number of dimensions (two, three). The specific figures included the smallest three possible symmetrical threedimensional matrices, $2 \times 2 \times 2,3 \times 3 \times 3,4 \times 4 \times 4$, and the two-dimensional matrices that best matched them for absolute number of units, $3 \times 3,5 \times 5,8 \times 8$. 


\section{EXPERIMENT 5}

\section{Method}

Subjects. Eight female and 4 male Oglethorpe University summer school students volunteered to participate.

Materials. The matrices were constructed of $16-\mathrm{cm}^{2}$ squares or $64-\mathrm{cm}^{3}$ wooden blocks, as in the previous experiments. There were six different matrices, and the matrix size was defined as small $(3 \times 3$, $2 \times 2 \times 2)$, medium $(5 \times 5,3 \times 3 \times 3)$, or large $(8 \times 8,4 \times 4 \times 4)$.

Thirty-two pathways were generated as in the previous experiments for each of the six matrices. Four tape recordings were prepared, each of which included eight trials with each matrix at each presentation rate. Two tapes began with all of the fast trials followed by all slow trials, and the other two were prepared in reverse order. Within each block of fast or slow presentations, trials were also blocked by matrix, with trial blocks of eight always beginning with small matrices and continuing with matrices alternating in dimensions and increasing in size. Specific pathways for each matrix appeared in fast and slow conditions on different tapes, and the order of matrices of differing numbers of dimensions was also counterbalanced across tapes. Each tape was played for 3 subjects.

Procedure. As each matrix was introduced for the first time, the task with respect to that matrix was explained and the subject was given two practice trials, in which the experimenter read the directional statements at a slow rate. Prior to every block of eight trials, the subject was given two practice trials with directions read at the appropriate rate on tape.

\section{Results}

The mean percent correct for each matrix is presented on the first two lines in Table 4 . A $3 \times 2 \times 2$ within-subject ANOVA showed significant main effects of presentation rate $\left[F(1,11)=38.56, M S_{\mathrm{e}}=2.18, p<.001\right]$ and matrix size $\left[F(2,22)=43.40, M S_{\mathrm{e}}=2.02, p<.001\right]$, and a significant interaction between rate and size $[F(2,22)$ $\left.=6.98, M S_{\mathrm{e}}=0.56, p<.005\right]$; but there was no significant effect of dimensionality, nor were any of the other interactions significant either. The comparison of the $3 \times 3 \times 3$ with the $5 \times 5$ matrix at slow speeds was significant by Bonferroni criteria at the .05 level.

Smaller numbers of subjects in this experiment make comparisons of high and low scorers somewhat tentative, yet nevertheless, the variability in overall performance allowed selection of 4 high scorers ( 2 of them female) with an average overall score of $87 \%$ correct and 5 low

Table 4

Mean Percent of Correct Trials for the Six Matrices in Experiment 5

\begin{tabular}{|c|c|c|c|c|c|c|}
\hline \multirow[b]{3}{*}{ Rate } & \multicolumn{6}{|c|}{ Matrix } \\
\hline & \multicolumn{2}{|c|}{ Small } & \multicolumn{2}{|c|}{ Medium } & \multicolumn{2}{|c|}{ Large } \\
\hline & $3 \times 3$ & $2 \times 2 \times 2$ & $5 \times 5$ & $3 \times 3 \times 3$ & $8 \times 8$ & $4 \times 4 \times 4$ \\
\hline \multicolumn{7}{|c|}{ All Subjects } \\
\hline Slow & 98 & 97 & 80 & 99 & 70 & 71 \\
\hline Fast & 89 & 84 & 69 & 66 & 48 & 45 \\
\hline \multicolumn{7}{|c|}{ High Scorers* } \\
\hline Slow & 100 & 100 & 91 & 100 & 88 & 88 \\
\hline Fast & 88 & 94 & 91 & 81 & 62 & 62 \\
\hline \multicolumn{7}{|c|}{ Low Scorers $\dagger$} \\
\hline Slow & 95 & 92 & 75 & 97 & 55 & 62 \\
\hline Fast & 85 & 70 & 52 & 45 & 30 & 25 \\
\hline
\end{tabular}

scorers (all female) with an average of $65 \%$ correct. The performance for these two subgroups by condition is presented on the final four lines of Table 4 . A $3 \times 2 \times 2 \times 2$ mixed ANOVA yielded significant main effects of group $\left[F(1,7)=16.76, M S_{\mathrm{e}}=4.74, p<.005\right]$, presentation rate $\left[F(1,7)=73.75, M S_{\mathrm{e}}=1.76, p<.001\right]$, and size $\left[F(2,14)=40.08, M S_{\mathrm{e}}=1.56, p<.001\right]$. Interactions were significant for group and size $\left[F(2,14)=4.54, M S_{\mathrm{e}}\right.$ $=1.56, p<.05]$, for group and presentation rate $[F(1,7)$ $\left.=6.97, M S_{\mathrm{e}}=1.16, p<.05\right]$, for size and presentation rate $\left[F(2,14)=9.07, M S_{\mathrm{e}}=2.21, p<.005\right]$, and for presentation rate and dimensionality $[F(1,7)=8.06$, $\left.M S_{\mathrm{e}}=0.78, p<.05\right]$. The three-way interaction of group, presentation rate, and size was also significant $\left[F(2,14)=5.42, M S_{\mathrm{e}}=0.41, p<.05\right]$.

Pathways in the $3 \times 3 \times 3$ matrix that included hidden blocks, as defined in the previous experiments, had an average of $81 \%$ correct trials; pathways with no hidden blocks had an average of $85 \%$ correct. Comparisons for the $4 \times 4 \times 4$ figure were more difficult because the majority of the pathways moved through at least 1 of the 18 blocks that could not be seen from the front, top, or side of the figure. One set of tapes, for example, included only one pathway with no hidden blocks. The other set of tapes, however, provided an interesting point of contrast. Four pathways in this set of 16 remained entirely on the front and top surfaces of the figure, whereas 3 remained in hidden blocks for 6 or 7 of the 8 pathway steps. Average performance was $75 \%$ and $72 \%$ accurate for the figures, respectively, against an average performance of $64 \%$ for all $4 \times 4 \times 4$ pathways in that tape set.

\section{Discussion}

The subjects' performance was sensitive to both matrix size and presentation rate. The interaction between presentation rate and size indicated that the detrimental effects of fast presentation rates were stronger for larger matrices. Performance with two- and three-dimensional matrices was roughly equivalent for matrices matched for total number of units, with one notable exception: performance with the $3 \times 3 \times 3$ matrix at the slow speed was significantly better than performance with the sizecomparable $5 \times 5$ matrix. At the fast rate, the $5 \times 5$ and $3 \times 3 \times 3$ matrices were roughly equivalent.

The results at the slow presentation rate based on all subjects provide a striking replication of Cornoldi et al.'s (1991) findings for subjects who had been selected because they were high visuospatial scorers. The pattern of performance across conditions is virtually identical, and overall performance levels are remarkably similar. This suggests that Cornoldi et al.'s sample of high visuospatial scorers, drawn from a population of high school students who presumably varied considerably in cognitive skills, was similar in ability to the sample reported here, which came from a relatively homogeneous cognitively skilled college population. It is unsurprising, therefore, that high and low scorers among our subjects at the slow rate showed no difference in patterns of performance, and relatively small differences in levels of performance. 
Overall, the performance of the present subjects at the fast presentation rates was similar in many respects to Cornoldi et al.'s (1991) results for subjects with low visuospatial skills. Scores for the present subjects at the fast rates showed the same pattern of accuracy as that of the low visuospatial scorers for all matrices except the $4 \times 4 \times 4$ matrix. Whereas Cornoldi et al. found large performance differences for low visuospatial scorers on the $8 \times 8$ and $4 \times 4 \times 4$ matrices, we found little difference between the two matrices even at fast rates for the lowest scoring subjects. The simplest explanation for these differences is that our college-student sample simply did not include subjects as low in visuospatial skills as Cornoldi et al.'s low visuospatial group.

\section{GENERAL DISCUSSION}

Comparisons of two- and three-dimensional processing in the matrix task can be based on one of two matching strategies: the number of units per dimension (e.g., $3 \times 3$ vs. $3 \times 3 \times 3$ ) or the approximate total number of units per matrix (e.g., $3 \times 3$ vs. $2 \times 2 \times 2$ ). Kerr (1987) argued that capacity is defined by units per dimension, based on nearly perfect performance with the two-dimensional $3 \times 3$ and three-dimensional $3 \times 3 \times 3$, but no larger matrix of either type. That claim has been supported here at slow presentation rates. When rates were increased, however, the drop in performance was disproportionately large for the $3 \times 3 \times 3$ matrix, and this was especially apparent for subjects who had difficulty with the imagery task in general. Although it is possible to keep track of 27 locational units distributed in a $3 \times 3 \times 3$ display, to do so apparently requires greater skill and longer processing times than does imagining the simpler $3 \times 3$ display.

The repeated comparison of the $2 \times 2 \times 2$ with the $3 \times 3$ figure presents a more complicated set of results. Subjects performed equally well with the two figures in Experiment 1 , but they selected a significantly slower presentation rate for the $2 \times 2 \times 2$, suggesting that the presentation rate was relevant to task performance for the two matrices. Comparison of the two figures at the fast presentation rates in subsequent experiments, however, failed to produce statistically reliable results. Although consistently in the direction of better performance for the $3 \times 3$, the comparisons were only significant in Experiments 3 and 4 . The failure to detect reliable differences may well have been due to ceiling effects, given the consistently high performance with these figures, even at fast presentation rates. If so, adding to task load by increasing pathway length or number of trials might facilitate detection of differences, as might the testing of subjects who have scored low on independent measures of visuospatial skills.

The division of subjects into high and low scorers on the basis of the total number of correct trials further substantiated Cornoldi et al.'s (1991) claim that individual differences play a large role in performance on the task described here. Differences in performance based both on the total number of units and on the number of spatial dimensions were much more apparent with low-than with high-scoring subjects.

There was no evidence in any of the experiments that pathways that moved through blocks that were occluded from the subject's viewpoint were more difficult than those that stayed in view, despite the fact that maintaining a point of view was critical to understanding the directions with respect to the figure. A change in vantage point would necessitate a translation of directional terms from the new vantage point to the old. The results, then, are more consistent with Neisser and Kerr's (1973) Gibsonian theory than with theories that preclude imagining aspects of the environment that are occluded from view (Keenan \& Moore, 1979).

In all of the experiments reported here, there is a natural confounding between the number of spatial dimensions in the matrices and the number of directional terms used to describe the pathways in the matrices. Four verbal terms describe pathways in two-dimensional matrices, whereas six terms are necessary to describe movements in three-dimensional matrices. This confound provides a source of alternative explanations for differences between the $3 \times 3$ and $3 \times 3 \times 3$ matrices at fast presentation rates. The simplest alternative explanation is that the verbal load of number of potential directional terms is the source of difficulty for three-dimensional figures. This possibility was addressed previously (Kerr, 1987) in an experiment that increased the verbal load for a $3 \times 3$ matrix to six terms by requiring that subjects keep track of three levels of changes in a nonspatial dimension (temperature) as well as position in the two-dimensional matrix. Performance on this task was significantly poorer than performance with the $3 \times 3 \times 3$ matrix, suggesting that the spatial dimensions and not the number of verbal terms was the relevant variable. A more sophisticated explanation based on the differences between verbal descriptions of two- and three-dimensional pathways was suggested to me by both J. I. Chumbley and C. Cornoldi in their reviews of this manuscript. According to this account, performance on the matrix task is mediated not by the number of spatial dimensions that must be maintained in a representational medium, but by the number of directions in which a pathway might move, given its current location. A pathway in a $2 \times 2$ matrix always has two directions of potential movement, whereas potential directions of movement in a $3 \times 3 \times 3$ matrix vary from six for the central block to three for the corner blocks. Thus, in the $3 \times 3$ matrix, if the randomly selected starting square is in one of the four corners, the directional uncertainty will be 2 ; if it is in one of the four squares on the middle of each side of the figure, the directional uncertainty will be 4 . The average directional uncertainty for this matrix, then, is 2.67 , or it would be if all steps of the pathway were randomly chosen. However, directional uncertainty is itself confounded with the probability of a unit's appearing in a pathway, given that the number of adjacent units affects both measures. For example, the probability that a given corner square will be the second step in a pathway that 
begins in a randomly selected starting square is half as great as the likelihood for the central square, because the central square has four "entry points" whereas a corner has only two. If the directional uncertainty of the squares in each matrix is "weighted" for the probability of appearing in a pathway on the basis of the number of entry points, the resulting theoretical estimates of the overall influence of directional uncertainty is 2.00 for the $2 \times 2$ figure in Experiment 1, 2.85 for the $3 \times 3$ figure, 3.00 for the $2 \times 2 \times 2$, and 4.13 for the $3 \times 3 \times 3$ figure. These estimates do not provide a strong predictive base for the preferred presentation rates of Experiment 1, but the results of the subsequent experiments do not render them implausible. The only finding that is clearly inconsistent with predictions based on this analysis is the equivalence of performance with the $8 \times 8$ and $4 \times 4 \times 4$ matrices in Experiment 5 . The influence of directional uncertainty may be more directly tested in future research by deliberately creating pathways in three-dimensional figures that differ on this measure, or by including a third set of directional terms for two-dimensional matrices (perhaps indicating moves in diagonal directions).

The results of the series of experiments reported here support neither of the theoretical positions that served as the focus for previous research centered on mental rotation tasks, at least in their simplest forms. The finding that fast presentation rates can be more disruptive when the stimuli in the imagery task are three-dimensional than when they are two-dimensional is inconsistent with theories that interpret imagery representation as fundamentally three-dimensional (Attneave, 1972; R. N. Shepard, $1981,1984)$. The equivalent performance with twodimensional stimuli that do and do not include the depth dimension contradicts the assumption of primacy for the picture plane in imagery representation (Kosslyn, 1980; Kosslyn \& Shwartz, 1977). Although these theories have been revised repeatedly, the focus has remained on viewer-centered imagery that includes a vantage point but no direct information about depth versus object-centered imagery with no particular point of view. Yet subjects in the research reported here were capable of maintaining a point of view while simultaneously keeping track of location in three-dimensional space. These results suggest that the nature of imagery processing is more complex than can be accounted for by either theoretical approach alone, and that the consideration only of these two positions may limit theoretical accounts unnecessarily.

\section{REFERENCES}

Atrneave, F. (1972). Representation of physical space. In A. W. Melton \& E. Martin (Eds.), Coding processes in human memory (Pp. 283306). Washington, DC: Winston.

Attneave, F., \& Curlee, T. E. (1983). Locational representation in imagery: A moving spot task. Journal of Experimental Psychology: Human Perception \& Performance, 9, 20-30.

Brooks, L. R. (1967). The suppression of visualization during reading. Quarterly Journal of Experimental Psychology, 40, 288-299.
Cornoldi, C., Cortesi, A., \& Preti, D. (1991). Individual differences in the capacity limitations of visuospatial short-term memory: Research on sighted and totally congenitally blind people. Memory \& Cognition, 19, 459-468.

Farah, M. J., Hammond, K. M., Levine, D. N., \& Calvanio, R. (1988). Visual and spatial mental imagery: Dissociable systems of representation. Cognitive Psychology, 20, 439-462.

Gibson, J. J. (1966). The senses considered as perceptual systems. Boston: Houghton Mifflin.

Gibson, J. J. (1979). The ecological approach to visual perception. Boston: Houghton Mifflin.

InTONS-PETERSON, M. J. (1983). Imagery paradigms: How vulnerable are they to experimenters' expectations? Joumal of Experimental Psychology: Human Perception \& Performance, 9, 394-412.

Jolicoeur, P., Regehr, S., Smith, L. B. J. P., \& Smith, G. N. (1985). Mental rotation of two-dimensional and three-dimensional objects. Canadian Journal of Psychology, 39, 100-129.

Just, M. A., \& Carpenter, P. A. (1985). Cognitive coordinate systems: Accounts of mental rotation and individual differences in spatial ability. Psychological Review, 92, 137-171.

KeEnan, J. M., \& MoORE, R. E. (1979). Memory for images of concealed objects: A reexamination of Neisser and Kerr. Journal of Experimental Psychology: Human Learning \& Memory, 5, 374-385.

KERR, N. H. (1987). Locational representation in imagery: The third dimension. Memory \& Cognition, 15, 521-530.

KerR, N. H., \& Neisser, U. (1983). Mental images of concealed objects: New evidence. Joumal of Experimental Psychology: Learning, Memory, \& Cognition, 9, 212-221.

Kosslyn, S. M. (1980). Image and mind. Cambridge, MA: Harvard University Press.

Kosslyn, S. M., SHWArtz, S. P. (1977). A simulation of visual imagery. Cognitive Science, 1, 265-295.

MetzleR, J., \& ShePARD, R. N. (1974). Transformational studies of the internal representation of three-dimensional objects. In R. L. Solso (Ed.), Theories in cognitive psychology: The Loyola Symposium (pp. 147-201). Potomac, MD: Erlbaum.

NeISSER, U., \& KERR, N. (1973). Spatial and mnemonic properties of visual images. Cognitive Psychology, 5, 138-150.

Peterson, L. R., Rawlings, L., Cohen, C. (1977). The internal construction of spatial patterns. In G. H. Bower (Ed.), The psychology of learning and motivation (Vol. 2. pp. 245-276). New York: Academic Press.

Peterson, M. J. (1975). The retention of imaged and seen spatial matrices. Cognitive Psychology, 7, 181-193.

Pinker, S. (1980). Mental imagery and the third dimension. Joumal of Experimental Psychology: General, 109, 354-371.

PINkER, S., Kosslyn, S. M. (1978). The representation and manipulation of three-dimensional space in mental images. Joumal of Mental Imagery, 2, 69-84.

ShePard, R. N. (1981). Psychophysical complementarity. In M. Kubovy \& J. R. Pomerantz (Eds.), Perceptual organization (pp. 279-341). Hillsdale, NJ: Eribaum.

SHEPARD, R. N. (1984). Ecological constraints on internal representation: Resonant kinematics of perceiving, imagining, thinking, and dreaming. Psychological Review, 91, 417-447.

ShePARD, R. N., \& CoOper, L. A. (1982). Mental images and their transformations. Cambridge, MA: MIT Press/Bradford Books.

SHEPARD, R. N., \& METZler, J. (1971). Mental rotation of threedimensional objects. Science, 171, 701-703.

ShePARD, S., \& Metzler, D. (1988). Mental rotation: Effects of dimensionality of objects and type of task. Journal of Experimental Psychology: Human Perception \& Performance, 14, 3-11.

ShWaRTZ, S. P. (1981). Three-dimensional mental rotation revisited: Picture plane rotation is really faster than depth rotation (Cognitive Science Tech. Rep. No. 9). New Haven, CT: Yale University, Cognitive Science Program.

(Manuscript received July 1, 1992; revision accepted for publication February 1, 1993.) 